\title{
Soluble L-selectin concentration in bronchoalveolar lavage fluid obtained from infants who develop chronic lung disease of prematurity
}

\author{
S Kotecha, M Silverman, R J Shaw, N Klein
}

\begin{abstract}
Aims-To explore the changes in neutrophil adhesion molecule expression and release into bronchoalveolar lavage fluid (BAL) obtained from infants who developed chronic lung disease (CLD).

Methods-BAL fluid was obtained from 37 infants: 18 (median gestation 26 weeks, birthweight $835 \mathrm{~g}$ ) who developed CLD, 12 ( 29 weeks, $1345 \mathrm{~g}$ ) with respiratory distress syndrome (RDS) and seven control infants (33 weeks, $2190 \mathrm{~g}$ ).

Results-Soluble L-selectin (sL-selectin) in BAL fluid from the CLD and non-CLD groups was similar immediately after birth, but in infants who subsequently developed CLD, sL-selectin remained persistently increased (at day 7: CLD 42.6 vs $\mathrm{RDS} 6.0 \mathrm{ng} / \mathrm{ml}, \mathrm{p}<0.05 ;$ CLD $v s$ controls $1.5 \mathrm{ng} / \mathrm{ml} ; \mathrm{p}<0.05)$. CD11b/CD18 expression on neutrophils obtained by BAL increased with time to reach a maximum at 17 days of age in infants who developed CLD.

Conclusions-These results suggest that leucocyte traffic persists in infants who develop CLD and may have an important part to play in the pathogenesis of CLD. (Arch Dis Child Fetal Neonatal Ed 1998;78:F143-F147)
\end{abstract}

Keywords: chronic lung disease of prematurity; adhesion molecules; bronchoalveolar lavage; neutrophils

Chronic lung disease of prematurity (CLD) remains a major cause of morbidity and mortality in preterm neonates. ${ }^{1}$ Although the risk factors of CLD are known, the mechanisms involved are still poorly understood. ${ }^{2}$ One consistent feature that has emerged in recent studies is evidence of pulmonary inflammation occurring between 7 and 10 days of age. ${ }^{3-11}$ Both neutrophils and alveolar macrophages have been implicated and their numbers are increased in CLD. ${ }^{356}$ Furthermore, concentrations of inflammatory cytokines such as tumour necrosis factor- $\alpha$, interleukin- $1 \beta$, interleukin- 6 and interleukin- 8 have been detected in lavage fluid from infants who develop CLD. ${ }^{4} 81011$

Leucocytes which mediate inflammation gain access to lung interstitium by migrating from the blood to the lung. This occurs as a result of interactions occurring between adhesion molecules on the surfaces of leucocytes and endothelial cells. ${ }^{12} 13$ The margination or "rolling" of leucocytes on the endothelial cell surface is predominantly mediated by selectins
(L-selectin on leucocytes and P- and Eselectins on endothelium) while firm adhesion and transmigration is largely mediated by integrins $\left(\beta_{1}\right.$ and $\left.\beta_{2}\right)$ and members of the immunoglobulin superfamily (ICAM-1 and -2).

Analysis of adhesion molecules on cells from the inflamed lungs of ventilated preterm infants is problematic. Biopsies are rarely performed and analysis of post mortem tissue contains artefacts inherent in such samples. For this reason, analysis of bronchoalveolar fluid has been used to provide information about the nature of the inflammatory response associated with acute lung injury. Most studies have concentrated on the cellular composition of BAL fluid. Now, however, the phenotype of aspirated cells can be examined in more detail. Soluble adhesion molecules can also be measured: soluble ICAM-1 is increased in many inflammatory disorders ${ }^{14} 15$ and sL-selectin (sL-selectin) is increased in serum from patients with leukaemia ${ }^{16}$ but decreased in serum from patients with adult RDS. ${ }^{17}$

Although neutrophils persist in lavage fluid from infants who develop CLD, little is known about the mechanisms involved in the migration of these cells to the lung in this disease. As part of a study to investigate this process in more detail, we examined BAL fluid from infants: (a) who developed CLD; (b) those developing and fully recovering from RDS; and (c) from control infants without severe lung injury. We assessed the concentration of sL-selectin in lavage fluid as well as the expression of L-selectin and the $\beta_{2}$ integrin, CD11b/ CD18, on inflammatory cells obtained in lung lavage fluid.

\section{Methods}

Three groups of mechanically ventilated infants who were admitted to the neonatal unit at Hammersmith Hospital were studied: (a) infants who developed CLD - those in whom a retrospective diagnosis was made on the basis of oxygen dependency at 28 days of age together with an abnormal chest $x$-ray picture ${ }^{18}$; (b) infants who developed and recovered from RDS - that is, were nursed in air and had a normal chest radiograph by day 28 of age; (c) control infants who received mechanical ventilation for non-respiratory reasons.

Infants of mothers who had either infection or prolonged rupture of membranes (of greater than 48 hours) were excluded as were infants with documented or suspected sepsis-that is, with positive blood cultures, blood films show- 
Table 1 Patient characteristics

\begin{tabular}{llll}
\hline & $C L D$ & $R D S$ & Controls \\
\hline Number & 18 & 12 & 7 \\
Gestation & $26(24-29)$ & $29(27-33)$ & $33(26-40)$ \\
Birthweight & $835(570-1080)$ & $1345(780-2130)$ & $2190(990-3600)$ \\
Male:female & $8: 10$ & $6: 6$ & $6: 1$ \\
Antenatal corticosteroids & $14(78 \%)$ & $5(42 \%)$ & $3(43 \%)$ \\
Surfactant treatment & $18(100 \%)$ & $11(92 \%)$ & $2(29 \%)$
\end{tabular}

Median (range) given for gestation and birthweight. CLD chronic lung disease of prematurity; RDS respiratory distress syndrome.

ing left shift of neutrophils ("toxic blood films"), or increased C-reactive protein $(>0.05$ $\mathrm{mg} / \mathrm{dl}$ ), at the time of entry. Infants who developed sepsis during the study were excluded if they had a positive blood culture or colonisation of endotracheal secretions. Informed consent was obtained from the parents to perform bronchoalveolar lavage of intubated infants receiving mechanical ventilation. The study was approved by the Hammersmith Hospital Ethics Committee.

Bronchoalveolar lavage was performed at the time of clinically indicated tracheal suctioning, as described before. ${ }^{4}{ }^{719}{ }^{20}$ With the baby lying supine, its head turned to the left, an FG 5 end-hole catheter was advanced through the end porthole of the endotracheal tube until resistance was felt. Two aliquots of $1 \mathrm{ml} / \mathrm{kg}$ (maximum $2 \mathrm{ml}$ ) of physiological saline were instilled and immediately after each aliquot a suction pressure of $7-8 \mathrm{kPa}(50 \mathrm{~mm} \mathrm{Hg})$ was applied to the catheter and the returned BAL fluid collected in a suction trap. Additional oxygen was given to maintain an oxygen saturation, as measured by a pulse oximeter, at 90-95\%. Infants were lavaged twice weekly for five weeks or until extubation, whichever occurred earlier. Thus infants who were extubated were excluded from further study as further lavage of these infants is unethical. For this reason, although the infants were lavaged twice weekly wherever possible, the concentration of sL-selectin was averaged if two samples were obtained after the first week of life.

The collected BAL samples were placed on ice and centrifuged at $500 \times g$ at room temperature for 10 minutes. After centrifugation the supernatant fluid was collected and stored at $-70^{\circ} \mathrm{C}$ until further analysis. Where possible the cells were resuspended in $1 \mathrm{ml}$ of physiological saline and several cytospins made using a cytocentrifuge (Shandon 3, Shandon Products Ltd, Cheshire), fixed in methanol and stored at $-70^{\circ} \mathrm{C}$ until immunocytochemistry for L-selectin and CD11b/CD18 was performed.

Soluble L-selectin was estimated in BAL fluid using a commercially available ELISA (R \& D Systems Europe Ltd, Oxon). The samples were assayed in duplicate and without knowledge of the sample origin. The lowest sensitivity of the ELISA was $0.72 \mathrm{ng} / \mathrm{ml}$.

The concentration of soluble L-selectin in lavage fluid was corrected for dilution by using urea dilution to estimate the epithelial lining fluid. The concentration of serum urea was measured using a urease method in a Technicon RA-XT discretionary analyser (Technicon Instruments Corp, Basingstoke). Urea concen- tration in lavage fluid was measured on the same analyser at an increased sensitivity whereby a linear dynamic range was achieved over $0.04-6.0 \mathrm{mmol} / \mathrm{l}$. The epithelial lining fluid was estimated by urea dilution: epithelial lining fluid as a proportion of total lavage fluid $=$ lavage fluid urea/serum urea. ${ }^{4-781920}$ The results for soluble L-selectin are expressed ng per $\mathrm{ml}$ of epithelial lining fluid.

Cytospins were incubated with monoclonal antibodies to L-selectin (CD62L, Serotec, Oxford) or CD $11 \mathrm{~b} / \mathrm{CD} 18$ (Coulter) for 1 hour at room temperature. After washing in phosphate buffered saline cytospins were incubated with a gold conjugated goat anti-mouse IgG (British Biocell International, Cardiff). Gold particles were detected by silver enhancement (British Biocell International, Cardiff) and visualised with light microscopy after counterstaining with Meyer's haematoxylin (BDH). IgG subclass matched controls were included for most patients. Two independent observers counted at least 50 neutrophils, identified morphologically, on each slide from a minimum of 10 high power fields, and a mean semiquantitative score from 0 (no staining) to 3 (strong staining) ascribed to each slide. The slides were assessed without prior clinical knowledge of the sample origins.

The concentration of sL-selectin was $\log$ transformed and represented as the geometric mean (SEM). Birthweight, gestation, and concentration of sL-selectin were compared between the three groups by using the KruskalWallis non-parametric ANOVA. Individual paired comparisons were made using the nonparametric Mann-Whitney U-test. $P<0.05$ was considered significant.

\section{Results}

The characteristics of the 37 infants studied are shown in table 1 . Among 30 requiring mechanical ventilation for RDS, 18 ( 8 boys and 10 girls) remained oxygen dependent at 28 days and had radiographical appearances suggestive of CLD, and 12 ( 6 boys, 6 girls) recovered from RDS and were nursed in air by 28 days of age. Seven control infants ( 6 boys, 1 girl) received mechanical ventilation for nonrespiratory reasons, including two for apnoea of prematurity, two with congenital muscular dystrophy, and three perioperatively for surgical disorders. When the gestational age and birthweight were compared, significant differences were found between the CLD and RDS (both $\mathrm{p}<0.001$ ); CLD and the control (both $\mathrm{p}<0.001$ ); and RDS and control groups (birthweight $\mathrm{p}<0.05$, gestation $\mathrm{p}>0.05)$. Fourteen mothers $(78 \%)$ had received antenatal corticosteroids in the CLD group, five (42\%) in the RDS group, and three (43\%) in the control group. All $18(100 \%)$ infants in the CLD group, $11(92 \%)$ of the 12 infants recovering from RDS, and two (29\%) of the seven infants in the control group had received two doses of surfactant (Curosurf, Serono Laboratories). In all cases where surfactant was given the first bronchoalveolar lavage was performed before the second dose of surfactant-at about 12-14 hours of age. The severity of respiratory failure 
as assessed by ventilatory requirements, including peak inspiratory pressures, mean airways pressure, oxygenation index, and inspired oxygen, at 12 hours of age, were similar for the CLD and RDS groups.

In infants who developed CLD, the mean concentration of sL-selectin was 35.5 (1.6) $\mathrm{ng} / \mathrm{ml}$ in the first BAL fluid obtained before the second dose of surfactant. It reached a maximum of $48.6(1.4) \mathrm{ng} / \mathrm{ml}$ on day 4 of age before declining gradually over the next 28 days to undetectable concentrations (fig 1). By contrast, in the RDS group the concentration of sL-selectin in the first BAL fluid obtained after birth was $22.3(1.7) \mathrm{ng} / \mathrm{ml}$, and this decreased rapidly to $6(3.9) \mathrm{ng} / \mathrm{ml}$ by 7 days of age. In the control infants the concentration of sL-selectin in lavage fluid was 36.6 (1.7) during the first few hours of age, reached a maximum of $82.6(1.3) \mathrm{ng} / \mathrm{ml}$ on day 4 , and rapidly declined to $<6 \mathrm{ng} / \mathrm{ml}$ thereafter until 28 days of age.

Soluble L-selectin in BAL fluid was significantly increased at 7 days of age in the CLD group compared with infants who did not progress to CLD $(p<0.01)$. This increase in soluble L-selectin at 7 days of age remained significant when the CLD group was compared with the RDS group $(p<0.05)$ or the control group $(p<0.05)$, but not when the RDS and control groups were compared $(\mathrm{p}>0.05)$.

Cytospins were prepared from cells obtained from 40 bronchoalveolar lavages and were stained for L-selectin and CD11b/CD18 by using immunocytochemistry. L-selectin was absent or present at low concentrations in most slides. Only control cells on days $1-4$ showed increased staining for L-selectin. In contrast, CD11b/CD18 was detected on the neutrophils present in most cytospins. In the CLD group a few cells frequently did not stain for either marker. It is unclear whether these cells had become non-viable before BAL fluid was aspirated. Because of the small number of samples collected, the infants who developed CLD were compared with those who did not (infants in the RDS and control groups). In infants who developed CLD, the relative staining for CD $11 \mathrm{~b} / \mathrm{CD} 18$ was 0.4 immediately after birth but increased and reached a maximum of 2.0 at day 17 before gradually declining to 0.5 by day

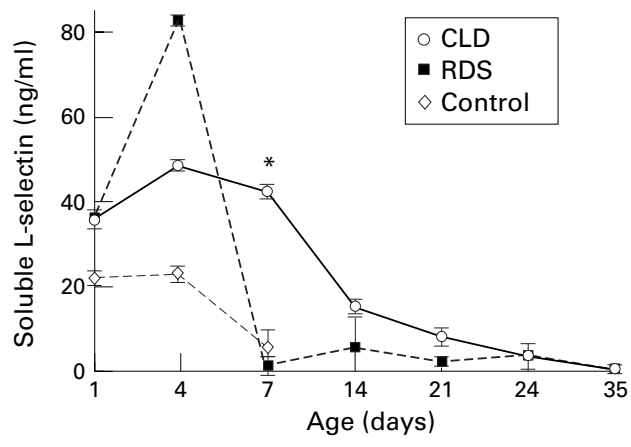

Figure 1 Concentration of soluble L-selectin (sL-selectin) in BAL fluid obtained from infants who developed $C L D$, recovered from $R D S$, and control infants ventilated for non-respiratory reasons. The concentration of sL-selectin is shown in $\mathrm{ng} / \mathrm{ml}$ of epithelial lining fluid which was estimated by urea dilution. Mean values (SEM) are shown. ${ }^{\star} p<0.05 C L D$ vs RDS, and CLD vs. controls.

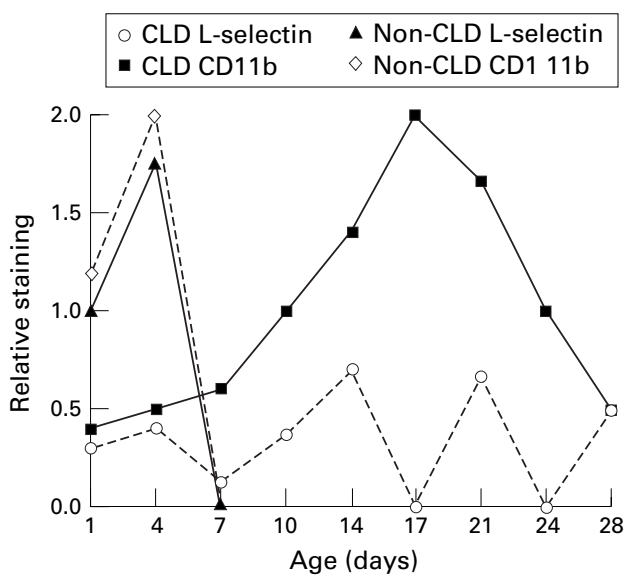

Figure 2 Semiquantitative representation of staining by immunocytochemistry for L-selectin and CD11b/CD18 of cells obtained from infants who developed CLD and infants who did not (infants with RDS and infants ventilated for non-respiratory reasons). Mean values are shown.

28 of age (fig 2). By contrast, in infants who were nursed in air by 28 days of age, the grading of staining was 1.2 immediately after birth. The staining reached a maximum of 2.0 on day 4 before rapidly declining to minimal staining by day 7 .

\section{Discussion}

We have observed changes in the expression of pulmonary leucocyte adhesion molecules in infants with CLD. CD11b/CD18, a ligand for ICAM-1, was increased on neutrophils obtained by bronchoalveolar lavage of infants who developed CLD when compared with those who did not. sL-selectin was also increased in bronchoalveolar lavage fluid obtained from all three groups but remained persistently high in infants who remained oxygen dependent and developed CLD.

Extravasation of leucocytes into the lung parenchyma and air spaces is mediated by adhesion molecules. ${ }^{12}{ }^{13}$ Cells initially roll on endothelial cells through selectins before transmigrating into the lung by utilising integrins and molecules of the immunoglobulin superfamily. The process of rolling seems to occur through the sequential binding and proteolytic cleavage of the selectins in a unidirectional manner. ${ }^{21}$ Cleaved L-selectin is biologically active and is then available to interact with surrounding cells such as leucocytes, endothelial, and epithelial cells.

The finding of sL-selectin in lung fluid indicates that cleavage of this molecule from leucocytes has occurred (fig 1) and may reflect leucocyte traffic into the lung. Its persistence in lavage fluid from the CLD group suggests that leucocytes continue to migrate from blood to the air spaces compared with those infants who did not progress to CLD. The low level of L-selectin staining seen on the surfaces of neutrophils in CLD would be consistent with L-selectin cleavage (fig 2). The initially high neutrophil L-selectin in the control group may reflect less extensive cleavage of this molecule in this group of babies. By contrast, the low expression of L-selectin on cells from the CLD group may be due to continuing pulmonary 
inflammation as there is some evidence to suggest that L-selectin expression may be downregulated on activation, at least in vitro. ${ }^{22}$ These observations are compatible with previous studies of acute lung inflammation in infants who develop CLD. ${ }^{2-11}$ More recent evidence, however, suggests that reduced shedding of L-selectin may increase neutrophil accumulation by decreasing rolling. ${ }^{23}$

Transmigration of neutrophils from the vasculature to the air spaces is mediated through $\beta_{2}$ integrins and their ligands ICAM-1 and ICAM-2. CD11b/CD18 is stored in the cytoplasmic granules of neutrophils and is translocated with CD18 as a heterodimer to the cell surface on stimulation . $\beta_{2}$ integrins and members of the immunoglobulin superfamily are upregulated by proinflammatory mediators, many of which also cause shedding of L-selectin. The increase in CD $11 \mathrm{~b} / \mathrm{CD} 18$ in the CLD group is therefore compatible with the L-selectin data, in that loss of L-selectin is observed with increased expression of CD11b/ CD18. It also supports our previously reported observation of increased sICAM in lavage fluid from infants who developed CLD. ${ }^{4}$

The measurement of adhesion parameters within lavage fluid must always be analysed with caution. Although increased expression of some adhesion molecules may suggest cellular activation, this may not always be the case. For example, CD11b/CD18 requires conformational change before it can bind to its ligand. Cells that are aspirated from the lungs are also non-adherent and therefore may not be representative of all cells present in the airways. This may explain why on day 1 there was apparently greater expression of CD $11 \mathrm{~b} / \mathrm{CD} 18$ on neutrophils from controls than in infants developing CLD. In the latter group activated neutrophils may be adherent to epithelial cells through the ligation of ICAM-1, leaving relatively non-activated cells free in lavage fluid. The free cells would be aspirated by the lavage procedure. For similar reasons the greatest expression of CD11b/CD18 may not actually represent the timing of maximal neutrophil activation. Soluble adhesion molecule concentrations may be misleading. For example, in the adult patients at risk of developing RDS, low concentrations of plasma sL-selectin were found. ${ }^{17}$ As biologically active selectin may be bound to endothelial cells, low concentrations of sL-selectin may not be an accurate reflection of leucocyte activation.

In contrast to endothelial-leucocyte interactions, migration of leucocytes across epithelium is less well understood. Although the interplay between $\beta_{2}$ - integrins and epithelial ICAM-1 has been established, the interactions between L-selectin and the epithelium are less clear. Our data suggest L-selectin-epithelium interactions may have a part to play in the recruitment of neutrophils from the blood to air spaces. A novel therapeutic strategy in the treatment of infants at risk of CLD would be to prevent migration of the neutrophils to the lung by the use of monoclonal antibodies directed to the adhesion molecules. Animal models based on experimental lung injury suggest that such antibodies may attenuate the histological lung injury when compared with untreated animals. ${ }^{24}{ }^{25}$ Furthermore, this reduction in lung injury is accompanied by a substantial decrease in neutrophil recruitment to the lungs of these treated animals. ${ }^{24}{ }^{25}$ However, such antibodies against adhesion molecules do not completely abolish the migration of neutrophils to the lung. This may be due to other non-adhesion molecule dependent mechanisms for neutrophil migration to the lung or due to inadequate access of the relevant antibody from the circulation to the site of adhesion molecule expression in the involved compartment.

In summary, our study has shown a persistent increase in sL-selectin in bronchoalveolar lavage obtained from infants who developed CLD and increased expression of neutrophil CD $11 \mathrm{~b} / \mathrm{CD} 18$ in this group of babies. The data support the hypothesis that active neutrophils increase expression of CD11b/CD18 as they enter the lung and at the same time surface L-selectin is shed, giving rise to increased sL-selectin in bronchoalveolar lavage fluid obtained from infants who develop CLD. In the wider context, these results provide further evidence that pulmonary inflammation is important in the pathogenesis of CLD.

We thank the parents and nurses at the Hammersmith Hospital Neonatal Unit, and $\mathrm{Mr}$ Jeremy Beecham, Department of Chemical Pathology, RPMS, for the measurement of urea in bronchoalveolar lavage fluid.

Dr S Kotecha was supported by a Medical Research Council Training Fellowship.

1 Currie AE, Kotecha S. Chronic lung disease of prematurity. Crit Care Med 1997;12:14-17.

2 Kotecha S. Cytokines in chronic lung disease of prematurity. Eur f Pediatr 1996;155:514-17.

3 Ogden BE, Murphy SA, Saunders GC, Pathak D, Johnson JD. Neonatal lung neutrophils and elastase/proteinase inhibitor imbalance. Am Rev Respir Dis 1984;130:817-21.

4 Kotecha S, Chan B, Azam N, et al. Increase in interleukin-8 and soluble intercellular adhesion molecule-1 in bronchoalveolar lavage fluid from premature infants who develop chronic lung disease. Arch Dis Child 1995;72: F90-6. 5 Arnon S, Grigg J, Silverman M. Pulmonary inflammatory
cells in ventilated preterm infants: effect of surfactant treatment. Arch Dis Child 1993;69:44-8.

6 Murch SH, Costeloe K, Klein NJ, MacDonald TT. Early increase of macrophage inflammatory protein $1 \alpha$ occurs in respiratory distress syndrome and is associated with poor respiratory distress syndrome and is
outcome. Pediatr Res 1996;40:490-7.

7 Kotecha S, Wilson L, Wangoo A, et al. Increase in

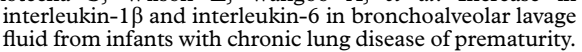
Pediatr Res 1996;40:250-6.

8 Kotecha S, Wangoo A, Silverman M, Shaw RJ. Increase in transforming growth factor- $\beta_{1}$ concentration in bronchoalveolar lavage fluid obtained from infants with chronic lung disease of prematurity. F Pediatr 1996;128:464-9.

9 Groneck P, Gotze Speer B, Oppermann M, Eiffert H, Speer CP. Association of pulmonary inflammation and increased microvascular permeability during the development of bronchopulmonary dysplasia: a sequential analysis of inflammatory mediators in respiratory fluids of high-risk preterm neonates. Pediatrics 1994;93:712-18.

10 Bagchi A, Viscardi RM, Taciak V, Ensor JE, McCrea KA, Hasday JD. Increased activity of interleukin- 6 but not tumor necrosis factor- $\alpha$ in lung lavage of premature infants is associated with the development of bronchopulmonary dysplasia. Pediatr Res 1994;36:244-52.

11 Murch SH, MacDonald TT, Wood CBS, Costeloe KL. Tumour necrosis factor in the bronchoalveolar lavage secretions of infants with the respiratory distress syndrome and the effect of dexamethasone treatment. Thorax 1992;47:44-7

12 Streiter RM, Lukacs NW, Standiford TJ, Kunkel SL. Cytokines and lung inflammation: mechanisms of neutrophil recruitment to the lung. Thorax 1993;48:764-9.

13 Symon FA, Wardlaw AJ. Selectins and their counter receptors: a bitter sweet attraction. Thorax 1996;51:1155-7.

14 Seth R, Raymond RD, Makgoba MW. Circulating ICAM-1 isoforms: diagnostic prospects for inflammatory and immune disorders. Lancet 1991;338:83-4. 
15 Shijubo N, Imai K, Shigehara K, Honda Y, Koba H, Tsujisaki $M$. Soluble intercellular adhesion molecule-1 (ICAM-1) in sera and bronchoalveolar lavage of patients with idiopathic fibrosis and p

16 Zetterberg E, Richter J. Correlation between serum levels of soluble L-selectin and leukocyte count in chronic myeloid and lymphocytic leukaemia and during bone marrow transplant. Eur f Haematol 1993:51;113-19.

17 Donnelly SC, Haslett C, Dransfield I, Robertson CE, Carter DC, Ross JA, et al. Role of selectins in development of adult respiratory distress syndrome. Lancet 1994;355:215-19.

18 Bancalari E, Abdenour GE, Feller R, Gannon J. Bronchopulmonary dysplasia: clinical presentation. $\mathcal{F}$ Pediatr 1979;95:819-23.

19 Grigg J, Arnon S, Silverman M. Fractional processing of sequential bronchoalveolar lavage fluid from intubated sequential bronchoalveolar lavage

20 Rennard SI, Ghafouri M, Thompson AB, Linder J, Vaughan
W, Jones K. Fractional processing of sequential bronchoalveolar lavage to separate bronchial and alveolar samples. Am Rev Respir Dis 1990;141:208-17.

21 Hogg N. Roll, roll, roll your leukocyte gently down the vein. Immunol Today 1992;13:113-15.

22 Haught WH, Mansour M, Rothlein R, Kishimoto TK, Mainolfi EA, Hendricks JB, et al. Alterations in circulating intercellular adhesion molecule-1 and L-selectin: further evidence for chronic inflammation in ischemic heart disease. Am Heart f 1996;132:1-8.

23 Walcheck B, Kahn J, Fisher JM, Wang BB, Fisk RS, Payan DG, et al. Neutrophil rolling altered by inhibition of L-selectin shedding in vitro. Nature 1996;380:720-3.

24 Doerschuk CM, Winn RK, Coxson HO, Harlan JM. CD18dependent and independent mechanisms of neutrophil emigration in the pulmonary and systemic microcirculation of rabbits. F Immunol 1990;144:2327-33.

25 Horgan MJ, Wright SD, Malik AB. Antibody against leukocyte integrin (CD18) prevents repurfusion-induced lung vascular injury. Am f Physiol 1990;259:L315-19. 